\title{
Expression of programmed cell death-1 and its ligand B7 homolog 1 in peripheral blood lymphocytes from patients with peripartum cardiomyopathy
}

\author{
Guozhi Xia ${ }^{1}$ | Xiaopu Zheng ${ }^{1}$ | Xinye Yao ${ }^{1}$ | Xiaowei Yao ${ }^{2}$ Zhongwei Liu ${ }^{2}$ | \\ Junkui Wang ${ }^{2}$
}

${ }^{1}$ Department of Cardiovascular Medicine, First Affiliated Hospital, Medical College, Xi'an Jiaotong University, Xi'an, China

${ }^{2}$ Department of Cardiovascular Medicine, Shaanxi Provincial People's Hospital, Xi'an, China

Corresponding Author

Xiaopu Zheng, MD, Department of

Cardiovascular Medicine, First Affiliated

Hospital, Medical College, Xi'an Jiaotong University, No. 277, Yanta West Road, Xi'an 710061, Shaanxi Province, China

Email: zhengxp00@163.com.
Background: Immune response has been postulated to play a prominent role in the pathogenesis of peripartum cardiomyopathy (PPCM). Given the importance of programmed death (PD)-1 and its ligand $\mathrm{B} 7$ homologue $1(\mathrm{~B} 7-\mathrm{H} 1)$ costimulatory molecules as an immune regulatory pathway, this study aimed to investigate the effect of PD-1 and B7-H1 expression on immune response in peripheral blood lymphocytes from the patients with PPCM.

Hypothesis: PD-1 and B7-H1 may be involved in modulating immune response in PPCM.

Methods: Peripheral blood lymphocytes were obtained from PPCM and pregnancy-matched healthy women. PD-1 and B7-H1 expression were determined using fluorescence quantitative reverse transcription-polymerase chain reactions (RT-PCR) and Western blot. The presence of serum interferon (IFN)- $\gamma$ and interleukin (IL)-4 were determined with enzyme-linked immunosorbent assay.

Results: The levels of pro-brain natriuretic peptide and IFN- $\gamma$ were markedly elevated, whereas the levels of left ventricular ejection fraction and IL-4 were significantly reduced in PPCM patients compared to controls. Additionally, both RT-PCR and Western blot revealed that the levels of PD-1 and B7-H1 expression were decreased significantly in PPCM patients compared with controls. A significant positive correlation was observed between PD-1 and B7-H1 expression. Furthermore, PD-1 and B7-H1 expression showed significant negative correlation with IFN- $\gamma$, as well as positive correlation with IL-4. Therefore, decreased expression of PD-1 and $\mathrm{B} 7-\mathrm{H} 1$ led to a dysregulating immune response such that cellular immunity linked to $\mathrm{T}$ helper (Th)1 cells was predominant over humoral immunity linked to Th2 cells in PPCM.

Conclusions: This study provided the first findings that PD-1 and B7-H1 expression were decreased, which might impair functional regulation of negative costimulation on immune response that may work in the etiopathogenesis of PPCM.

\section{KEYWORDS}

Costimulation, PD-1, B7-H1, Immune, Peripartum, Cardiomyopathy

\section{1 | INTRODUCTION}

Peripartum cardiomyopathy (PPCM) is an idiopathic cardiomyopathy that is characterized by symptoms and signs of heart failure associated with decreased left ventricular systolic function and significant morbidity and mortality, occurring between the commencement of the last month of pregnancy and the end of the fifth month in the postpartum period, in women without preexisting symptoms, sign, or history of heart disease. ${ }^{1}$ The pathogenesis of PPCM remains uncertain, but immune response has been proposed. ${ }^{2}$ During pregnancy it is conceptualized to be a T helper type 2 (Th2) phenomenon, implying that the maternal immune response is shifted toward humoral immunity dominance linked to Th2 cells as a result of the bidirectional interaction between mother and fetus. Consequently, increased immunoglobulin (Ig) synthesis and decreased cellular response are associated with the maintenance of pregnancy. The enhancement of 
T helper type 1 (Th1) cells causes immunoregulation imbalance resulting in detrimental effects on cardiac function. This abnormal immune response may contribute to initiating of the pregnancy-associated cardiomyopathy. ${ }^{3}$

Programmed death (PD)-1 and its ligand B7 homologue I (B7$\mathrm{H} 1$ ), the important members of the CD28 costimulatory factors superfamily, have been well-known as a crucial negative costimulatory molecules and play a critical role in modulating immune response for the favorable immunological environment of a successful pregnancy. ${ }^{4}$ Several studies have shown that the engagement of the PD1 with B7-H1 depressed $T$ helper (Th) cell-mediated immune reactivity through reducing expression and secretion of multiple cytokines. $^{5}$ Encouraged by these studies, we analyzed the effect of PD-1 and $\mathrm{B} 7-\mathrm{H} 1$ expression on immune response in peripheral blood lymphocytes from a cohort of PPCM patients to provide new insights into the pathogenesis of PPCM.

\section{2 | METHODS}

\section{1 | Patients and healthy controls}

From October 2013 to November 2015, patients ( $n=38$ ) admitted to our hospital were enrolled in the study and newly diagnosed with PPCM according to the definition proposed in a recent position paper from the Heart Failure Association of the European Society of Cardiology Working Group ${ }^{3}$ : (1) age between 18 and 37 years, (2) development of congestive heart failure in the last month of pregnancy or within the first 5 months of delivery, (3) absence of recognizable heart disease before pregnancy, (4) no other identifiable cause for cardiac failure, and (5) left ventricular (LV) systolic dysfunction demonstrated by echocardiographic analysis such as depressed LV ejection fraction (LVEF) (LVEF < 0.55). Exclusion criteria were: (1) significant organic valvular heart disease, (2) gestational hypertension and preeclampsia, (3) metabolic disorders such as diabetes mellitus and thyroid disease, and (4) incomplete clinical data. Pregnancymatched healthy women with confirmed normal cardiac function (echocardiography, LVEF $\geq 0.55$ ) were invited to participate in the study as controls $(n=38)$. All patients provided informed consent according to a protocol approved by the ethics committee of First Affiliated Hospital of Xi'an Jiaotong University.

\subsection{Clinical assessments and blood tests}

Clinical assessments such as demographics, onset of symptoms and signs during the first presentation, heart rate, blood pressure, echocardiography, and diseases in pregnancy were obtained from the patients at baseline.

Peripheral blood was extracted from all the subjects in a fasting state in the following morning of the admission day. Blood specimens were collected in sterile tubes containing ethylenediaminetetraacetic acid as an anticoagulant. Plasma and serum were separated by centrifugation (Eppendorf, Hamburg, Germany) at $1500 \mathrm{~g}$ for 10 minutes. Aliquots were stored at $-80^{\circ} \mathrm{C}$ for future analysis. Laboratory workup was performed as routine investigation by hospital laboratories for hemoglobin, alanine transaminase, aspartate transaminase, creatinine, creatine kinase isoenzyme, troponin I, and pro-brain natriuretic peptide (proBNP). The concentrations of serum interferon (IFN)- $\gamma$ and interleukin (IL)-4 were measured with commercially available enzymelinked immunosorbent assay (ELISA) kits according to the manufacturer's recommendations (Elabscience Biotechnology, Wuhan, China). The concentration results were calculated based on the absorbance value and standard curves. The intra-assay and interassay variation coefficients for both ELISA measurements were $<10 \%$.

\section{3 | Isolation of the peripheral blood lymphocytes}

There were $10 \mu \mathrm{L}$ of venous blood from each subject that were mixed with an equal volume of phosphate buffered saline (PBS) $(8.0 \mathrm{~g}$ $\left.\mathrm{NaCl}, 0.2 \mathrm{~g} \mathrm{KCl}, 0.24 \mathrm{~g} \mathrm{KH}_{2} \mathrm{PO}_{4}, 3.628 \mathrm{~g} \mathrm{Na}_{2} \mathrm{HPO}_{4} \times 12 \mathrm{H}_{2} \mathrm{O}, \mathrm{pH} 7.4\right)$ in a tube. There were $20 \mu \mathrm{L}$ of diluted blood carefully layered in another $50-\mu \mathrm{L}$ tube. Next, the tubes were then centrifuged at $2000 \mathrm{~g}$ for 10 minutes at room temperature. After centrifugation, the lymphocytes formed a distinct band at the interface between the sample layer and the lymphocyte separation medium (human) (Li Rui Biotechnology, Shanghai, China). A pipette was used to transfer the lymphocytes suspension into a centrifuge tube without removing the upper layer. An equal volume of PBS was added into the centrifuge tube containing the lymphocyte suspensions and were mixed by gently inverting the tubes until the lymphocytes were homogenously diluted. Subsequently, the tubes were centrifuged at $4000 \mathrm{~g}$ for $2 \mathrm{~min}$ utes. Supernatants were discarded, and the lymphocytes were resuspended in PBS and centrifuged again at 4000g for 2 minutes. Supernatants were discarded, and an equal volume of PBS was added to each tube and mixed gently to dislodge the lymphocytes and bring them to a homogenous suspension. Then, purified peripheral blood lymphocytes were collected and added to a sterile tube for the studies described next.

\section{4 | Reverse transcription-polymerase chain reactions assay}

Total RNA was extracted from peripheral blood lymphocytes with Trizol reagents (Invitrogen, Carlsbad, CA), through centrifugation for 1 minute at $12000 \mathrm{~g}$. After confirming RNA concentration and assessment of purity with ultraviolet absorbance at $260 \mathrm{~nm}$ on a Nanodrop 1000 spectrophotometer (Thermo Fisher Scientific, Waltham, MA), equal amounts of total RNA from each sample were reversely transcribed into cDNA using a reverse transcription kit (Takara, Kusatsu, Japan) in accordance with the manufacture's instructions. Equal amounts of cDNA for each sample were used as template for fluorescence quantitative reverse transcriptionpolymerase chain reactions (RT-PCR). The following sequencespecific primers were designed by using Primer Premier 5.0 software (PREMIER Biosoft, Palo Alto, CA) for PCR amplification: (1) PD-1 primers, 5'-GCA CGA GGG ACA ATA GGA-3' (forward) and 5'-GAC AAT GGT GGC ATA CTC-3' (reverse); (2) B7-H1 primers, 5'-CAG GGC ATT CCA GAA AGA-3' (forward) and 5'-CCT CCA TTT CCC AAT AGA C-3' (reverse); (3) the housekeeping gene $\beta$-actin primers, 5'-CCT GGG CAT GGA GTC CTG TG-3' (forward) and 5'-TCT TCA 
TTG TGC TGG GTG CC-3' (reverse). Thermal cycling was performed with an S1000 Thermal Cycler PCR detection system (Bio-Rad, Hercules, CA). Each reaction contained $3 \mu \mathrm{L}$ of $\mathrm{cDNA}, 0.96 \mu \mathrm{L}$ of primer, $3 \mu \mathrm{L}$ diethylpyrocarbonate and $6 \mu \mathrm{L}$ Taq polymerase. The protocol included an initial denaturation step at $95^{\circ} \mathrm{C}$ for 3 minutes, followed by 40 cycles of 10 seconds at $95^{\circ} \mathrm{C}, 30$ seconds at $57^{\circ} \mathrm{C}$, and $30 \mathrm{sec}-$ onds at $72^{\circ} \mathrm{C}$, followed by 5 minutes at $72^{\circ} \mathrm{C}$. Fluorescence signals were collected at $60^{\circ} \mathrm{C}$. Each sample was assayed in triplicate and compared to arbitrary values assigned to standard melt curves generated for the target gene to obtain relative abundance of amplified products. These values were then normalized to those of $\beta$-actin.

\subsection{Western blot assay}

Western blot was used to monitor PD-1 and B7-H1 proteins expression in peripheral blood lymphocytes. Briefly, following fractionation by $12 \%$ sodium dodecyl sulfate-polyacrylamide gel electrophoresis, cells were electrophoretically transferred to a nitrocellulose membrane. After blocking in $5 \%$ nonfat dry milk, the membranes were incubated with primary antibodies against PD-1 and B7-H1 (goat anti-human PD-1, goat anti-human B7-H1 [Everest Biotech, Oxford, UK]; mouse anti-human $\beta$-actin [ZSGB-BIO, Beijing, China]) at 1:1000 dilution overnight at $4{ }^{\circ} \mathrm{C}$, and then washed twice with PBS containing 0.1\% Tween-20. Subsequently, bands were visualized with horseradish peroxidase-conjugated secondary antibodies at 1:2000 dilution (rabbit anti-goat IgG and rabbit anti-mouse IgG [ZSJQ-BIO]) for 60 minutes at $37^{\circ} \mathrm{C}$. Protein expression levels were normalized using $\beta$-actin. The relative protein levels were determined with the Gel Imaging Analysis System (BioRad).

\section{6 | Statistical analysis}

Database management and statistical analyses were performed with SPSS software (version 13.0; IBM, Armonk, NY). Statistical graphs were applied to GraphPad Prism 5 (GraphPad Software, La Jolla, CA). Continuous variables were expressed as mean \pm standard deviation. Comparisons of means between PPCM patients and controls were assessed using an independent samples $t$ test for normally distributed variables. A Wilcoxon rank sum test was used if data were not normally distributed. Categorical variables were displayed as frequencies (percentages) and compared with the $\chi^{2}$ test. The Spearman correlation coefficient was calculated to disclose relationships of PD-1 and B7-H1 expression with Th cytokines. A $P$ value $<0.05$ was considered a significant difference.

\section{3 | RESULTS}

\section{1 | Baseline clinical profiles of study subjects}

The levels of LVEF and proBNP, indicators of the severity of cardiac dysfunction, were diminished and elevated in PPCM patients, respectively. Statistical analysis showed both PPCM patients and controls differed significantly in LVEF, proBNP, and creatinine (Figure 1A-C). All other clinical data between the 2 groups that displayed no significant difference were presented in Table 1.
Additionally, the concentration of serum IFN- $\gamma$ in PPCM patients tended to be significantly higher than that in controls $(189.3 \pm 42.8$ $\mathrm{pg} / \mathrm{mL}$ vs $161.7 \pm 37.3 \mathrm{pg} / \mathrm{mL}, P=0.004$; Figure $1 \mathrm{D})$. Furthermore, the serum level of IL-4 was significantly lower in PPCM patients compared with controls $(79.8 \pm 17.4 \mathrm{pg} / \mathrm{mL}$ vs $88.7 \pm 18.7 \mathrm{pg} / \mathrm{mL}$, $P=0.036$; Figure 1E).

\section{2 | Expression of PD-1 and B7-H1 in peripheral blood lymphocytes}

The mRNA and protein expression levels of PD-1 and B7-H1 in peripheral blood lymphocytes were significantly lower in PPCM patients than those in controls (Figures 2 and 3). The peripheral blood lymphocytes from PPCM patients showed decreased expression of PD-1 and B7-H1 mRNA compared with controls (PD-1: $0.65 \pm 0.15$ vs $0.74 \pm 0.17, \mathrm{~B} 7-\mathrm{H} 1: 0.67 \pm 0.16$ vs $0.76 \pm 0.16$ ) The relative expressions of PD-1 and $\mathrm{B} 7-\mathrm{H} 1$ mRNA in peripheral blood lymphocytes from patients with PPCM were significantly lower than that in controls (PD-1: $P=0.018$, Figure $2 A$; $B 7-\mathrm{H} 1$ : $P=0.015$, Figure 2B).

To further verify PD-1 and B7-H1 expression, Western blot was applied to evaluate levels of the protein expression. As expected, these results were consistent with fluorescence quantitative RT-PCR. Under $\beta$-actin protein, PD-1 and $\mathrm{B} 7-\mathrm{H} 1$ protein bands were more distinct in PPCM patients compared to controls (Figure $3 A, C$ ). The quantitative analysis results revealed that the level of PD-1 protein expression was observed to be decreased markedly in peripheral blood lymphocytes from patients with PPCM compared with controls $(0.62 \pm 0.09$ vs $1.05 \pm 0.09$, $P<0.001$, Figure 3B), similar to the level of $B 7-\mathrm{H} 1$ protein expression between the 2 groups $(0.78 \pm 0.07$ vs $1.01 \pm 0.06$, $P<0.001$, Figure 3D).

Consequently, it was reasoned that peripheral blood lymphocytes poorly expressed PD-1 and B7-H1 associated with immunoregulation imbalance according to changes of IFN- $\gamma$ and IL-4 in PPCM patients.

\section{3 | Correlates of PD-1 and B7-H1 expression with Th cytokines}

Cytokines are considered to play an important role in immune regulation. We thus investigated the correlation of PD-1 and B7-H1 expression with Th cytokines, including Th1 cell-derived IFN- $\gamma$, which is seen predominantly in cellular-immune and Th2 cell-derived IL-4, which facilitates humoral immunity. ${ }^{6}$

In the study population ( $\mathrm{n}=76$ ), we discovered a significant positive correlation between PD-1 and B7-H1 expression (Figure 4A,B). For further correlation of PD-1 and B7-H1 expression with Th cytokines, our study indicated there was a significant negative correlation observed between IFN- $\gamma$ and PD-1 and B7-H1 expression (Figure 4C-F), but significant positive correlation between IL-4 and PD-1 and B7-H1 expression (Figure 4G-J). Therefore, it seemed that decreased expression of PD-1 and B7-H1 was associated with enhancement of Th1 cell activity. 
(A)

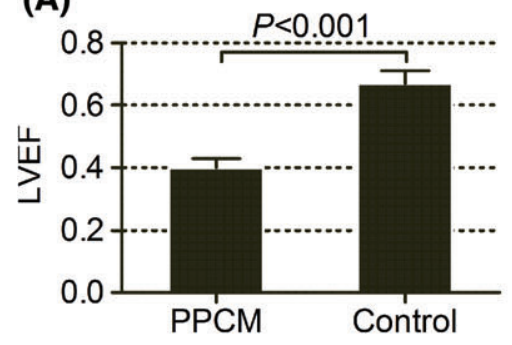

(B)

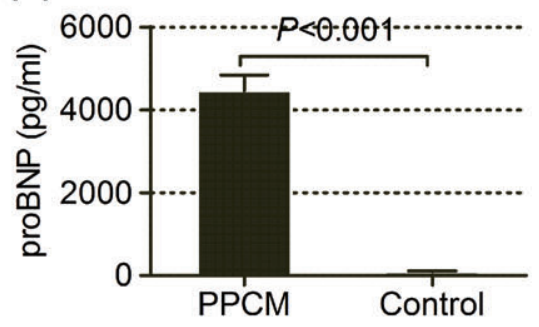

(C)

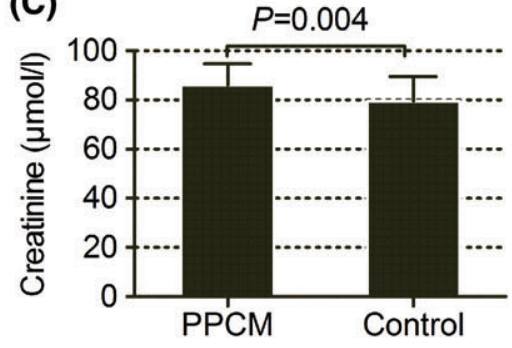

(D)

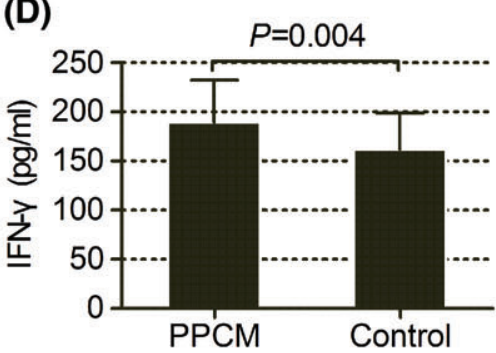

(E)

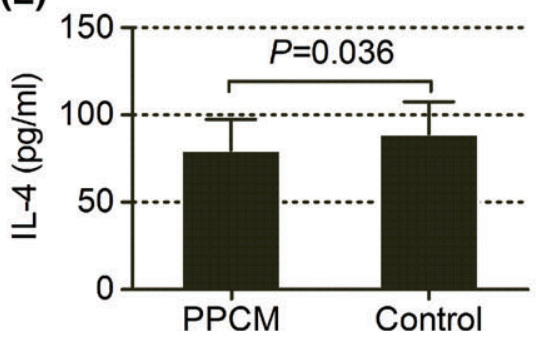

FIGURE 1 Comparison of baseline clinical profiles between peripartum cardiomyopathy (PPCM) (n = 38) and pregnancy-matched controls ( $n=38$ ). Abbreviations: IFN, interferon; IL, interleukin; LVEF, left ventricular ejection fraction; proBNP, pro-brain natriuretic peptide.

\section{4 | DISCUSSION}

The etiology of PPCM remains unknown; many potential causes have been proposed, including viral myocarditis, nutritional deficiencies, microchimerism, inflammation, and oxidative stress. A recent advance

TABLE 1 Baseline clinical profiles of the study population

\begin{tabular}{|c|c|c|c|}
\hline Variable & $\begin{array}{c}\text { PPCM } \\
\text { Patients }(n=38)\end{array}$ & $\begin{array}{l}\text { Controls } \\
(n=38)\end{array}$ & $\begin{array}{c}P \\
\text { Value }\end{array}$ \\
\hline Age (y) & $27 \pm 4$ & $28 \pm 5$ & 0.305 \\
\hline HR (beats/min) & $86 \pm 10$ & $82 \pm 8$ & 0.088 \\
\hline BMI $\left(\mathrm{kg} / \mathrm{m}^{2}\right)$ & $21.4 \pm 2.3$ & $22.2 \pm 3.2$ & 0.267 \\
\hline $\begin{array}{l}\text { Multiple } \\
\text { gestation }\end{array}$ & $2(5.3)$ & $2(5.3)$ & 1.000 \\
\hline Primigravidas & $30(78.9)$ & $32(84.2)$ & 0.554 \\
\hline $\mathrm{SBP}(\mathrm{mm} \mathrm{Hg})$ & $118 \pm 12$ & $117 \pm 9$ & 0.710 \\
\hline DBP (mm Hg) & $77 \pm 6$ & $75 \pm 6$ & 0.083 \\
\hline $\mathrm{RBC}\left(\times 10^{12} / \mathrm{L}\right)$ & $4.06 \pm 0.66$ & $4.03 \pm 0.48$ & 0.863 \\
\hline $\mathrm{Hb}(\mathrm{g} / \mathrm{L})$ & $111.03 \pm 9.67$ & $114.05 \pm 9.46$ & 0.173 \\
\hline WBC $\left(\times 10^{9} / L\right)$ & $8.34 \pm 1.87$ & $8.45 \pm 1.68$ & 0.795 \\
\hline $\operatorname{PLT}\left(\times 10^{9} / \mathrm{L}\right)$ & $243.43 \pm 26.62$ & $234.50 \pm 35.59$ & 0.219 \\
\hline FBG (mmol/L) & $4.79 \pm 0.77$ & $4.93 \pm 0.70$ & 0.400 \\
\hline HbA1c (\%) & $5.23 \pm 0.52$ & $5.15 \pm 0.51$ & 0.506 \\
\hline ALT (IU/L) & $35 \pm 8$ & $33 \pm 7$ & 0.313 \\
\hline AST (IU/L) & $35 \pm 7$ & $33 \pm 6$ & 0.248 \\
\hline CK-MB (U/L) & $10 \pm 3$ & $8 \pm 3$ & 0.075 \\
\hline Albumin (g/L) & $33.51 \pm 1.68$ & $34.33 \pm 2.82$ & 0.129 \\
\hline $\begin{array}{l}\text { Troponin I } \\
\text { (ng/mL) }\end{array}$ & $0.03 \pm 0.01$ & $0.02 \pm 0.01$ & 0.241 \\
\hline hs-CRP (mg/L) & $10.40 \pm 3.24$ & $10.42 \pm 3.18$ & 0.981 \\
\hline
\end{tabular}

Abbreviations: ALT, alanine transaminase; AST, aspartate transaminase CBMI, body mass index; DBP, diastolic blood pressure; FBG, fasting blood glucose; $\mathrm{Hb}$, hemoglobin; $\mathrm{HbA} 1 \mathrm{c}$, hemoglobin $\mathrm{A} 1 \mathrm{c} ; \mathrm{HR}$, heart rate; hs CRP, high sensitive C-reactive protein; CK-MB, creatine kinase isoenzyme-MB; PLT, platelets; PPCM, peripartum cardiomyopathy; RBC, red blood cell; SBP, systolic blood pressure; WBC, white blood cell. in the understanding of the pathogenetic basis of this disorder has proposed that abnormal immune response may be the important molecular pathway underlying pregnancy-induced cardiomyopathy. ${ }^{7}$ Some clinicians regarded PPCM as a facultative autoimmune disorder, which was relatively acute and organ specific compared to other chronic systemic autoimmune disorders. ${ }^{8}$ Autoantibodies against normal cardiac tissue proteins that were not present in the sera of patients with idiopathic cardiomyopathy have been identified to play an important role in the pathogenesis of PPCM. ${ }^{9,10}$ Studies have shown that elevated autoantibodies correlated with increased LV dimensions and reduced LV systolic function, thereby suggesting a diagnostic and prognostic value of these autoantibodies. ${ }^{11,12}$ Consistent with these findings, this study revealed PPCM patients with a lower LVEF and a higher plasma level of proBNP compared with healthy controls as the result of reduced LV systolic function.

The activation of T lymphocytes requires 2 signals, 1 of which is delivered by the $\mathrm{T}$ cell receptor complex after antigen recognition, and the other that requires the engagement of costimulatory receptors. The second signal can be either positive, which leads to full $T$ cell activation, or negative, which can downregulate immune responses. In regard to negative $\mathrm{T}$ cell signaling, the inhibitory costimulatory receptor molecule PD-1 and its ligand, $\mathrm{B} 7-\mathrm{H} 1$ has been shown to play a role in regulating immune response. PD-1 has been shown to be constitutively expressed by double negative thymocytes, natural killer cells, and its expression can be induced on activated CD4 and CD8 T cells, B cells, and monocytes. B7-H1 is expressed on antigen-presenting cells following cellular activation or exposure to several cytokines. B7-H1 has also been found on a subpopulation of activated $\mathrm{T}$ cells. In addition, B7-H1 can be constitutively expressed or induced by a variety of parenchymal or endothelial cells. In the present study, peripheral blood lymphocytes were chosen as the research tissue because abnormalities in various biochemical, hemorheological, and immunological elements in systematic diseases can be examined using them, and the method of isolating lymphocytes from 
FIGURE 2 Expression of programmed death (PD)-1 and B7 homologue 1 (B7-H1) mRNA. Representative histograms on analysis show the relative expression levels of PD-1 mRNA (A, $P=0.018$ ) and $B 7-\mathrm{H} 1$ mRNA ( $B, P=0.015)$ in peripheral blood lymphocytes between peripartum cardiomyopathy (PPCM) $(n=38)$ and controls $(n=38$ ) by fluorescence quantitative reverse transcriptionpolymerase chain reactions.
(A)

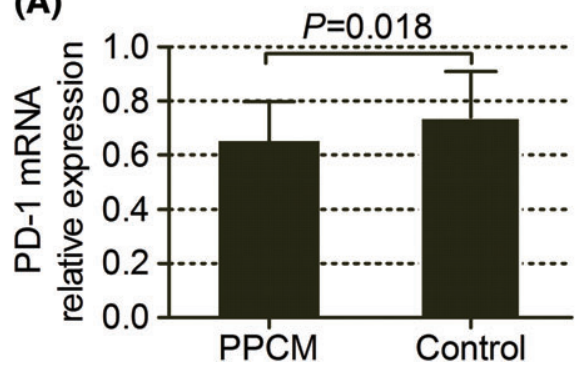

(B)

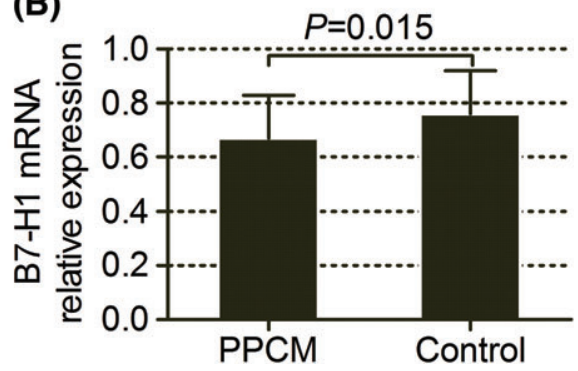

peripheral whole blood was acceptable. Moreover, the enrolled subjects in the control group were pregnancy-matched healthy women with confirmed normal cardiac function to reduce the impact of pregnancy on the results. We first determined the expression of PD-1 and $\mathrm{B} 7-\mathrm{H} 1$ in peripheral blood lymphocytes from patients with PPCM. The significant changes in the expression of PD-1 and B7-H1 were observed in this study, which suggested that decreased expression of PD-1 and B7-H1 may be involved in dysregulating immune response and work in the development and progression of PPCM.

To the best of our knowledge, it is generally accepted that pregnancy is established and maintained by the balance between cellular and humoral immunity. Th1 and Th2 cells' immunity alterations with a shift toward humoral immunity dominance linked to Th2 cells contribute to the amelioration homeostasis of the maternal immune system and maintenance of pregnancy. ${ }^{13}$ Th cells can be divided into subtypes according to their prevalent profile of cytokines production. IFN- $\gamma$, primarily secreted from Th1 cells, mediates delayed hypersensitivity and drives cellular immunity. In addition, IFN- $\gamma$ stimulates the ThO cells to differentiate into Th1 cells capable of secreting inflammatory cytokines including tumor necrosis factor- $\alpha$ and IL-1. Therefore, Th1 cells are seen predominantly in cellular immune responses. Likewise, IL-4, mainly produced by Th2 cells, causes antibody producing and participates in allergic-type responses. If the Th0 cells are exposed to IL-4, then they develop into Th2 cells that facilitate humoral immunity. ${ }^{14,15}$

PD-1 has been defined as a negative regulator of the immune response, limiting the activation of $B$ and $T$ cells, and protecting
(A)

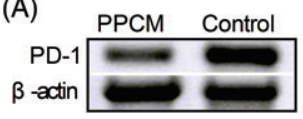

(C)

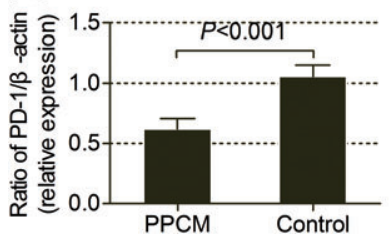

(B)

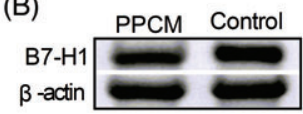

(D)

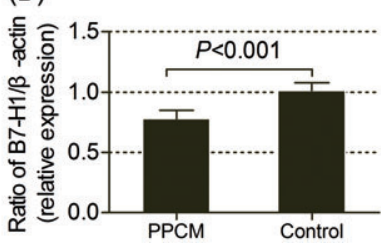

FIGURE 3 Expression of programmed death (PD)-1 and B7 homologue 1 (B7-H1) proteins. (A, C) Representative immunoreaction bands of PD-1 and B7-H1 proteins measured using Western blot. Representative histograms on analysis revealed the relative expression levels of $\mathrm{PD}-1$ protein $(\mathrm{B}, \mathrm{P}<0.001)$ and $\mathrm{B} 7-\mathrm{H} 1$ protein $(D, P<0.001)$ were reduced markedly in peripheral blood lymphocytes from peripartum cardiomyopathy (PPCM) $(n=38)$ compared with controls $(n=38)$. against autoimmunity. ${ }^{16} \mathrm{PD}-1$ is predominantly expressed on activated $\mathrm{T}$ and $\mathrm{B}$ cells. B7-H1, a member of B7 family of cosignaling molecules, is mainly expressed on immune cells including $B$ cells, $T$ cells, and macrophages. ${ }^{17}$ The binding of PD-1 to B7-H1 expressed on activated $\mathrm{T}$ cells transmits a signal that regulates the balance among Th1 cells inhibition and Th2 cells activation, and subsequently cytokines secretion, to maintenance of the Th2 phenomenon in healthy peripartum women via its phosphorylation effect on the downstream signaling molecules, including phosphatidylinositol 3-kinase and serine (PI3K), threonine protein kinase (Akt), and phosphatidylinositol 3-triphosphate (PIP3). ${ }^{18-21}$ The result of our study was in concordance with the concept about regulation of negative costimulation of PD-1 and B7-H1 on Th1/Th2 balance. This study provides the findings that PD-1 and $\mathrm{B} 7-\mathrm{H} 1$ expressions were decreased, which might inhibit the phosphorylation effect on the PI3K/PIP3/Akt signaling pathway, resulting in impairing functional suppression of Th1 cells activation. Therefore, the process enhanced Th1 cells activation and Th1 cytokines secretion, and limited Th2 cells activation and Th2 cytokines secretion in PPCM patients. ${ }^{22,23}$ Accordingly, our results in this study showed that there was a markedly increased IFN- $\gamma$ level and a significantly decreased IL-4 level in PPCM, but reversed the results in normal pregnant females, implying that IFN- $\gamma$ produced by Th1 cells predominate over IL-4 derived from Th2 cells in PPCM. ${ }^{24,25}$ The analysis of cytokines was recognized as an important tool for describing the condition of Th1/Th2 balance. ${ }^{10,23}$ These data added further credibility to the view that immunologic regulation imbalance existed in patients with PPCM.

Our results must be carefully explained with great caution. There were some limitations in the present study. First, PPCM is a diagnosis by exclusion, because some of the subjects were pregnant women, coronary angiography and catheterization were not allowed for application to exclude other kinds of cardiac diseases. Second, owing to no specific funding available, the case number was insufficient, as only 38 peripartum women were enrolled in the study. Third, the underlying mechanism that PD-1 and B7-H1 mediated the delicate balance between Th1 and Th2 cells was not clear. Consequently, further studies are needed.

\section{5 | CONCLUSION}

The present study investigated the expression of PD-1 and B7-H1 in peripheral blood lymphocytes from patients with PPCM. Our results 
(A)

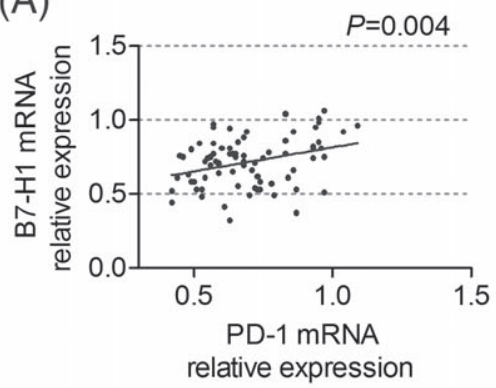

(D)

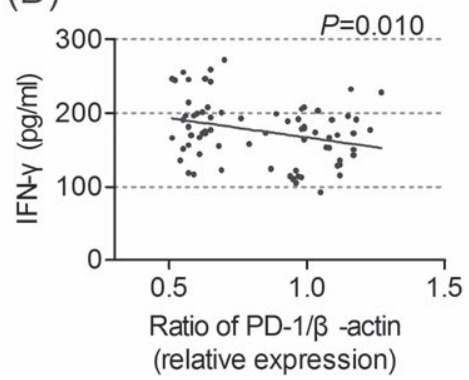

(G)

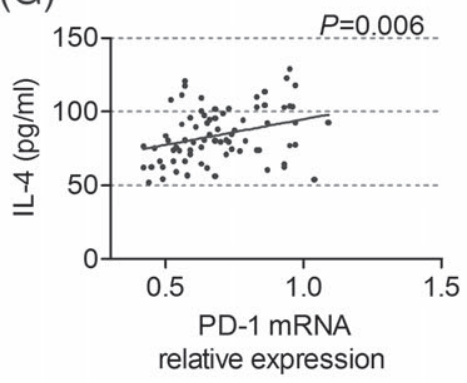

(B)

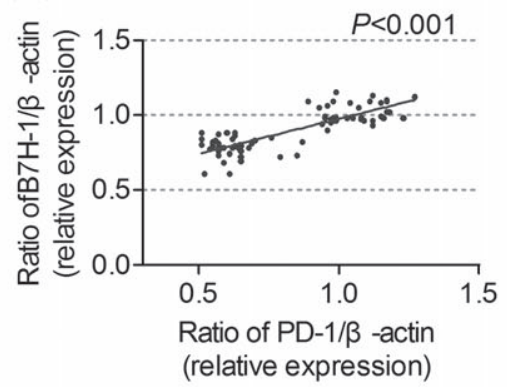

(E)

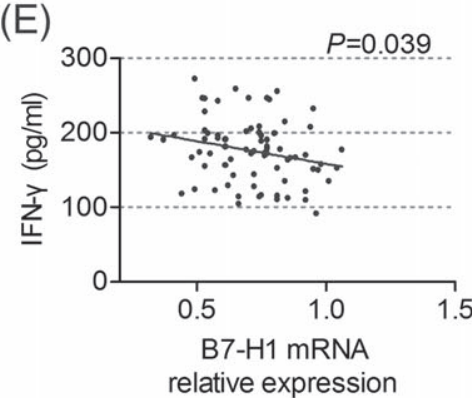

(H)
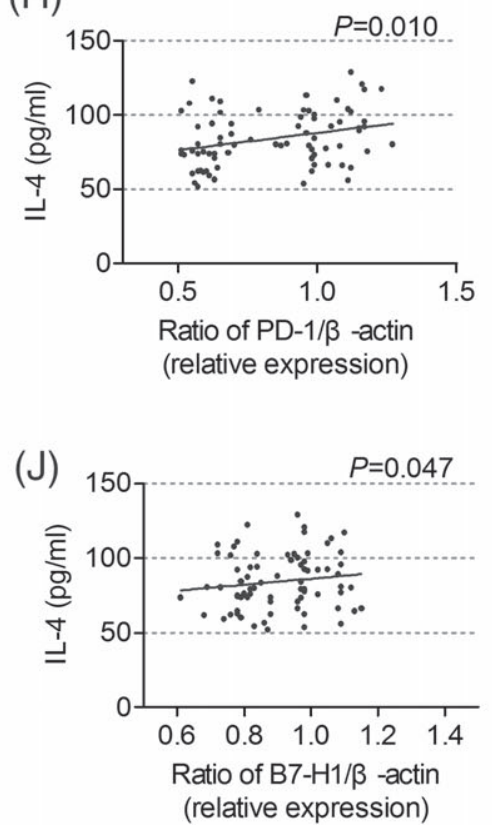

(C)

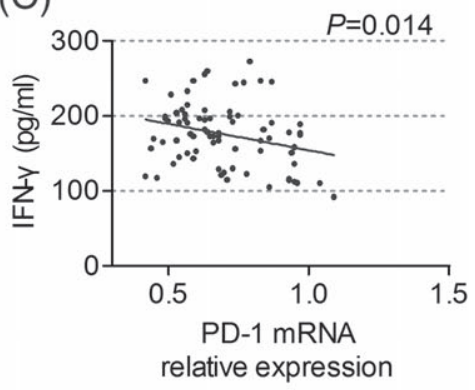

(F)

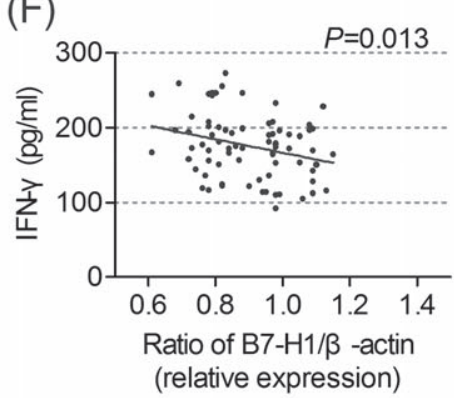

(I)

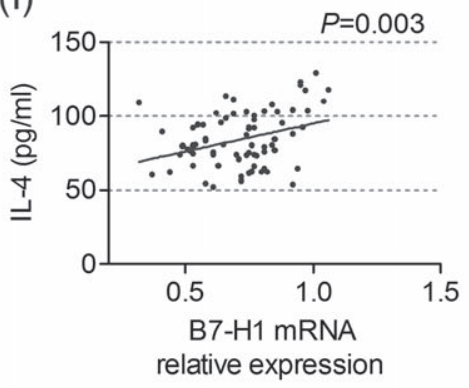

FIGURE 4 Correlates of programmed death (PD)-1 and B7 homologue 1 (B7-H1) expression with $\mathrm{T}$ helper cytokines in the study population $(n=76)$. Significant positive correlation was analyzed between PD-1 and B7-H1 expression (A, B) and interleukin (IL)-4 (G, H), also between B7$\mathrm{H} 1$ expression and IL-4 (I, J), but significant negative correlation was analyzed between interferon (IFN)- $\gamma$ and PD-1 expression (C, D) and B7-H1 expression (E, F).

revealed that markedly elevated IFN- $\gamma$ produced by Th1 cells negatively correlated with $\mathrm{PD}-1$ and $\mathrm{B} 7-\mathrm{H} 1$ expression, significantly decreased IL-4 produced by Th2 cells positively correlated with PD-1 and $\mathrm{B} 7-\mathrm{H} 1$ expression. In addition, these data added further credibility to the view that decreased expression of PD-1 and B7-H1 was associated with Th1/Th2 imbalance, and reversed the immunologic condition that cellular immunity linked to Th1 cells was predominant over humoral immunity linked to Th2 cells in PPCM. This study provided the first findings that PD-1 and B7-H1 expression was decreased, which might impair functional regulation of negative costimulation on cellular immunity that may work in the etiopathogenesis of PPCM.

\section{ACKNOWLEDGMENTS}

The authors acknowledge and thank Xiaowei Yao, Zhongwei Liu, and Junkui Wang of Shaanxi Provincial People's Hospital for their assistance in this study (PD-1 and B7-H1 expression determined using RT-PCR and Western blot). 


\section{1 | Conflict of interests}

The authors declare no potential conflict of interests.

\section{REFERENCES}

1. Garq J, Palaniswamy C, Lanier GM. Peripartum cardiomyopathy: definition, incidence, etiopathogenesis, diagnosis, and management. Cardiol Rev. 2015;23:69-78.

2. Adar T, Grisaru-Granovsky S, Ben Ya'acov A, Goldin E, Bar-Gil Shitrit A. Pregnancy and the immune system: general overview and the gastroenterological perspective. Dig Dis Sci. 2015;60:2581-2589.

3. Sliwa K, Hilfiker-Kleiner D, Petrie MC, et al. Current state of knowledge on aetiology, diagnosis, management, and therapy of peripartum cardiomyopathy: a position statement from the Heart Failure Association of the European Society of Cardiology Working Group on peripartum cardiomyopathy. Eur J Heart Fail. 2010;12:767-778.

4. Poole JA, Claman HN. Immunology of pregnancy. Implications for the mother. Clin Rev Allergy Immunol. 2004;26:161-170.

5. Gianchecchi E, Delfino DV, Fierabracci A. Recent insights into the role of the PD-1/PD-L1 pathway in immunological tolerance and autoimmunity. Autoimmun Rev. 2013;12:1091-1100.

6. Vanaki E, Ataei M, Sanati MH, et al. Expression patterns of Th1/Th2 transcription factors in patients with guttate psoriasis. Acta Microbiol Immunol Hung. 2013;60:163-174.

7. Haghikia A, Kaya Z, Schwab J, et al. Evidence of autoantibodies against cardiac troponin I and sarcomeric myosin in peripartum cardiomyopathy. Basic Res Cardiol. 2015;110:60-72.

8. Sundstrom JB, Fett JD, Carraway RD, Ansari AA. Is peripartum cardiomyopathy an organ-specific autoimmune disease? Autoimmun Rev. 2002;1:73-77.

9. Sliwa K, Skudicky D, Bergemann A, Candy G, Puren A, Sareli P. Peripartum cardiomyopathy: analysis of clinical outcome, left ventricular function, plasma levels of cytokines and Fas/APO-1. J Am Coll Cardiol. 2000;35:701-705.

10. Forster O, Hilfiker-Kleiner D, Ansari AA, et al. Reversal of IFNgamma, oxLDL and prolactin serum levels correlate with clinical improvement in patients with peripartum cardiomyopathy. Eur J Heart Fail. 2008;10:861-868.

11. Warraich RS, Sliwa K, Damasceno A, et al. Impact of pregnancyrelated heart failure on humoral immunity: clinical relevance of G3subclass immunoglobulins in peripartum cardiomyopathy. Am Heart J. 2005;150:263-269.

12. Fett JD, Ansari AA. Inflammatory markers and cytokines in peripartum cardiomyopathy: a delicate balance. Expert Opin Ther Tarqets. 2010;14:895-898.

13. Chaouat G, Ledee-Bataille N, Dubanchet S, Zourbas S, Sandra O, Martal J. TH1/TH2 paradigm in pregnancy: paradigm lost? Cytokines in pregnancy/early abortion: reexamining the $\mathrm{TH} 1 / \mathrm{TH} 2$ paradigm. Int Arch Allergy Immunol. 2004;134:93-119.

14. Hirahara K, Vahedi G, Ghoreschi K, et al. Helper T-cell differentiation and plasticity: insights from epigenetics. Immunology. 2011;134: 235-245.

15. Paulus WJ. How are cytokines activated in heart failure? Eur J Heart Fail. 1999;1:309-312.

16. Fife BT, Bluestone JA. Control of peripheral T-cell tolerance and autoimmunity via the CTLA-4 and PD-1 pathways. Immunol Rev. 2008;224:166-182.

17. Chen L, Zhang Z, Chen W, et al. B7-H1 up-regulation on myeloid dendritic cells significantly suppresses $T$ cell immune function in patients with chronic hepatitis B. J Immunol. 2007;178:6634-6641.

18. Francisco LM, Sage PT, Sharpe AH. The PD-1 pathway in tolerance and autoimmunity. Immunol Rev. 2010;236:219-242.

19. Keir ME, Butte MJ, Freeman GJ, Sharpe AH. PD-1 and its ligands in tolerance and immunity. Annu Rev Immunol. 2008;26:677-704.

20. Parry RV, Chemnitz JM, Frauwirth KA, et al. CTLA-4 and PD-1 receptors inhibit T-cell activation by distinct mechanisms. Mol Cell Biol. 2005;25:9543-9553.

21. Okazaki T, Maeda A, Nishimura H, Kurosaki T, Honjo T. PD-1 immunoreceptor inhibits $\mathrm{B}$ cell receptor-mediated signaling by recruiting src homology 2-domain-containing tyrosine phosphatase 2 to phosphotyrosine. Proc Natl Acad Sci U S A. 2001;98:13866-13871.

22. Ansari AA, Fett JD, Carraway RE, Mayne AE, Onlamoon N, Sundstrom JB. Autoimmune mechanisms as the basis for human peripartum cardiomyopathy. Clin Rev Allergy Immunol. 2002;23:301-324.

23. Sliwa K, Förster O, Libhaber E, Fett JD, Sundstrom JB, HilfikerKleiner D, Ansari AA. Peripartum cardiomyopathy: inflammatory markers as predictors of outcome in 100 prospectively studied patients. Eur Heart J. 2006;27:441-446.

24. Saito S, Sakai M, Sasaki Y, Tanabe K, Tsuda H, Michimata T. Quantitative analysis of peripheral blood Th0, Th1, Th2 and the Th1:Th2 cell ratio during normal human pregnancy and preeclampsia. Clin Exp Immunol. 1999;117:550-555.

25. Wegmann TG, Lin H, Guilbert L, Mosmann TR. Bidirectional cytokine interactions in the maternal-fetal relationship: is successful pregnancy a TH2 phenomenon? Immunol Today. 1993;14:353-356.

How to cite this article: Xia G, Zheng X, Yao X, Yao X, Liu Z and Wang J. Expression of programmed cell death-1 and its ligand B7 homolog 1 in peripheral blood lymphocytes from patients with peripartum cardiomyopathy. Clin Cardiol. 2017;40:307-313. https://doi.org/10.1002/clc.22661 